\title{
Structures de médiation et intégration des immigrants haïtiens à Paris
}

\section{Margarita Mooney}

Traducteur : Sabine Erbès-Seguin

\section{CpenEdition \\ Journals}

\section{Édition électronique}

URL : https://journals.openedition.org/remi/4300

DOI : $10.4000 /$ remi.4300

ISSN : $1777-5418$

\section{Éditeur}

Université de Poitiers

\section{Édition imprimée}

Date de publication : 1 avril 2008

Pagination : 89-114

ISBN : 978-2-911-627-48-2

ISSN : 0765-0752

\section{Référence électronique}

Margarita Mooney, "Structures de médiation et intégration des immigrants haïtiens à Paris ", Revue européenne des migrations internationales [En ligne], vol. 24 - $n^{\circ} 1$ | 2008, mis en ligne le 01 avril 2011 consulté le 14 avril 2022. URL : http://journals.openedition.org/remi/4300 ; DOI : https://doi.org/ 10.4000/remi.4300

Ce document a été généré automatiquement le 14 avril 2022.

(c) Université de Poitiers 


\title{
Structures de médiation et intégration des immigrants haïtiens à Paris
}

\author{
Margarita Mooney \\ Traduction : Sabine Erbès-Seguin
}

1 Plus de 200 ans après que la révolution haïtienne eut aboli le système colonial français à Saint Domingue, instabilité politique et misère économique ont incité des milliers de Haïtiens à émigrer en France ${ }^{1}$. Pourtant, on sait peu de choses sur les voies d'entrée et les modèles d'insertion des quelque 25000 Haïtiens qui vivent en France métropolitaine. Dans cet article, je présente les chiffres collectés au printemps 2003, alors que je préparais mon doctorat en sociologie-une étude comparative des influences religieuses sur l'insertion des immigrants haïtiens à Miami, Montréal et Paris. Parmi les trois pays sur lesquels a porté ma recherche, la France paraît offrir un accueil très favorable aux immigrants : selon le modèle républicain français, il suffit de devenir citoyen français pour faire passer au second plan toutes les autres différences, race, éducation, culture.

2 En 2005, la crise des banlieues - où résident beaucoup d'immigrés - porta à nouveau les défis posés par l'intégration des immigrants au cœur du débat national. Cependant, en tant que chercheuse américaine étudiant l'immigration en France peu d'années avant cette crise, j'avais constaté que beaucoup des intellectuels avec qui je discutais restaient fermement ancrés dans l'idéologie républicaine, même lorsque je leur faisais part de mes observations et des données qui semblaient contredire l'égalité promise par le modèle républicain. Les données quantitatives et qualitatives, collectées en 2003 et que je présente ici, montrent à l'évidence que les immigrants haïtiens en France ne deviennent pas impossibles à différencier des Français, que ce soit en termes de culture ou de position socio-économique. Pourtant, ce type d'analyse et les conclusions tirées de ces données sont souvent occultés par une ferme croyance en l'idéologie républicaine. À la suite des émeutes de 2005, de nombreux chercheurs français et des observateurs américains des tendances et politiques d'immigration en France ont 
réclamé plus de données sur l'intégration des immigrants en France et une discussion renouvelée, qui dépasse les limites de l'idéologie républicaine.

Bien que je me concentre sur un seul groupe d'immigrants - les Haïtiens - cet article entend cependant contribuer à combler les limites théoriques et empiriques de nos connaissances sur l'intégration des immigrants en France. Je présente tout d'abord des chiffres recueillis à partir de nombreuses sources gouvernementales françaises. Ils font apparaître les voies par lesquelles les Haïtiens sont entrés en France, les quartiers où ils s'installent, ainsi que les types d'emploi qu'ils occupent. Prises dans leur ensemble, ces données montrent que, en raison du nombre limité de moyens légaux d'entrée en France, de leur faible niveau d'éducation et de leur concentration dans les banlieues de Paris, qui rend problématique leur insertion au sein de la société française, les Haïtiens doivent mener un combat pour atteindre le même niveau que les Français de naissance. En second lieu, les entretiens que j'ai menés avec des immigrants haïtiens, ainsi que l'observation d'évènements religieux et sociaux, vont jusqu'à suggérer que les groupes intermédiaires et leurs responsables servent souvent de médiateurs entre les immigrants et l'État. Pourtant, l'idéologie républicaine française, et l'ensemble des politiques publiques $-\mathrm{y}$ compris sur la laïcité à la française - qu'elle a induit, négligent le fait que les immigrants font souvent confiance aux structures intermédiaires dans leur processus complexe d'intégration. Quoiqu'il ne soit pas possible dans le cadre limité de cet article d'établir des comparaisons avec les modèles d'insertion des Haïtiens aux États-Unis et au Canada, mon raisonnement selon lequel les structures intermédiaires contribuent à l'intégration des immigrants provient sans aucun doute de mon immersion dans l'expérience vécue par les immigrants haïtiens aux États-Unis et au Canada ${ }^{2}$. La relative indifférence de l'État français à ces structures de médiation prive ici les immigrants haïtiens d'un cadre d'insertion par ailleurs important dans d'autres pays.

\section{Le modèle républicain français}

Le modèle républicain français met l'accent sur une identité nationale basée sur la citoyenneté, il encourage les immigrants à substituer à leur identité nationale les valeurs et la culture françaises, et rejette toute représentation politique fondée sur l'origine nationale (Horowitz, 1992; Lamont, 2000). Ce modèle d'insertion diverge de ceux que l'on rencontre dans d'autres pays où existent de nombreuses communautés haïtiennes. Par exemple, le melting pot américain et le multiculturalisme canadien comportent à la fois une diversité ethnique et une représentation politique basée sur la race ou l'ethnie.

5 Bien que le modèle républicain soit le plus souvent défini comme une idéologie, il a influencé les structures légales et politiques françaises de plusieurs façons, par exemple, en ne prenant pas en compte les catégories ethniques et raciales dans les recensements. Ainsi, et bien que la diversité ethnique se soit accrue en France, « racial and ethnic identities... have not been generally understood as legitimate political, or even statistical categories $»^{3}$ (Lieberman, 2001, p. 35). De nombreux politiciens et commentateurs politiques dans le débat sur l'immigration arguent que la France doit se protéger des effets désintégrateurs du modèle de melting pot culturel des États-Unis, du pluralisme structurel des groupes ethniques, de la mobilisation politique sur une base ethnique et de la ségrégation résidentielle des immigrants (Kastoryano, 1996). 
6 En relation étroite avec le modèle républicain, le principe de laïcité à la française ${ }^{4}$ avait pour objectif de retirer aux Églises établies leur rôle dans les politiques et les services sociaux. En général, aux États-Unis, les associations privées, y compris religieuses, jouent un rôle important dans l'octroi de services sociaux, alors qu'en France, en raison du principe de laïcité, c'est l'État-providence qui est progressivement devenu le principal fournisseur de ces services (Esping-Andersen, 1990).

\section{Haïti de l'indépendance aux migrations en France}

7 Dans quelle mesure l'idéologie républicaine et la notion de laïcité à la française facilitent-ils notre compréhension de l'intégration contemporaine des immigrants haïtiens en France? En premier lieu, la Révolution haïtienne de 1804 contredit, de plusieurs façons, l'idée selon laquelle les colonies françaises tireraient des avantages réels des idéaux d'égalité universelle de la Révolution et du modèle républicain français. L'action des esclaves haïtiens était fondée sur la conviction que langue et culture continueraient à les séparer de leurs colonisateurs à moins d'obtenir une indépendance totale par rapport à la France, et même si leur était octroyée la citoyenneté française, ce qui se produisit finalement dans d'autres colonies françaises des Caraïbes, comme la Guadeloupe et la Martinique. La Constitution de la nouvelle nation haïtienne rejeta également le principe de laïcité à la française, par lequel la République française privait l'Église catholique d'une grande partie de son influence. Bien que les relations entre Haïti et le Vatican aient été assez tendues juste après la Révolution, un Concordat rétablit, en 1860, de bons rapports, et la Constitution haïtienne octroya à l'Église catholique des droits et privilèges spéciaux, notamment en ce qui concernait l'éducation (Nérestant, 1994). C'est pourquoi les Haïtiens immigrant en France sont en général préoccupés par les divisions entre races et entre classes dans et entre les nations, et présument que l'Église et l'État devraient coopérer en matière d'éducation et de sécurité sociale.

Actuellement, l'intégration des Haïtiens en France s'inscrit dans les débats en cours sur la citoyenneté et l'égalité. Au cours des années 1980, lorsqu'un grand nombre de Haïtiens commença à arriver en France, les banlieues parisiennes revenaient de manière récurrente dans le débat public pour illustrer l'échec de l'intégration des immigrants (Body-Gendrot, 2000 ; Noiriel, 1986). Le gouvernement leur avait apporté de nombreuses aides, par exemple en matière de logement social, mais la récession et la désindustrialisation réduisirent les opportunités offertes aux immigrés sur le marché du travail. Des parallèles commencèrent à être faits entre les projets gouvernementaux de logement social dans les banlieues des grandes villes, comme Paris, Lyon et Marseille, et les ghettos américains - qui se caractérisaient par des taux de criminalité élevés, par la délinquance juvénile et les violences à l'école (Body-Gendrot, 2000).

Bien que les transformations économiques soient largement responsables du chômage et de la dégradation des relations sociales dans les banlieues, une partie de la société française, autour de Jean-Marie Le Pen et du Front national, prétendait que les immigrés, notamment les Nord-Africains, étaient trop différents des Français sur le plan culturel pour pouvoir être intégrés. Des enquêtes d'opinion publique ont confirmé l'existence d'une perception selon laquelle certains immigrés seraient culturellement trop différents pour pouvoir s'intégrer (Hargreaves, 1995). 


\section{Structures de médiation et intégration des immigrants}

10 Afin de mieux centrer le débat sur les expériences d'immigration, j'utilise le concept de structures de médiation. Cette notion est plus souvent employée dans la littérature américaine que dans la littérature française sur l'intégration des immigrants. Des travaux, classiques aux États-Unis sur la théorie de l'immigration, tels que Polish Peasant in Europe and America, deThomas et Znaniecki (1927) estimaient que la religion était le fondement des communautés ethniques et considéraient que les institutions religieuses étaient des moyens indispensables à l'intégration des immigrants. D'autres auteurs, comme Oscar Handlin dans The Uprooted: the Epic Story of the Great Migrations that Made the American People (Handlin, 1951), de même que Milton Gordon dans Assimilation in American Life: the Role of Race, Religion and National Origins (Gordon, 1964) ont étendu la thèse de Thomas et Znaniecki sur les structures de médiation à d'autres groupes d'immigrants et à des associations tant religieuses qu'ethniques. Par exemple, Gordon a décrit comment "the immigrant subsociety mediates between the native culture of the immigrant and the American culture. The recognition of this fact is the indispensable prerequisite for the effective use of the communication channels and influence networks of the immigrants' communal life to aid and encourage the achievement of worthwhile acculturation goals $\|^{5}$ (1964, p. 244).

11 À mon sens, le modèle républicain français ne précise pas comment les immigrants font l'apprentissage de la culture et des modes de vie des Français d'origine. La conservation de liens étroits avec d'autres immigrés y est perçue comme un frein à l'intégration. Un autre point de vue, tel que proposé par les auteurs cités ci-dessus, soutient que les communautés d'immigrés constituent des médiations indispensables entre les nouveaux arrivants et les populations d'origine. Par exemple, Gordon déclare que l'État doit reconnaître ces structures de médiation et coopérer avec elles pour atteindre l'objectif d'intégration des immigrés.

12 Le concept de structures de médiation apparaît aussi dans le débat sur la société civile et l'État providence. Berger et Neuhaus, observateurs critiques de l'État providence américain, définissent les structures de médiation comme "those institutions standing between the individual in his private life and the large institutions of public life ${ }^{6}$ $(2000$, p. 144). Très critiques vis-à-vis des modalités d'octroi des services sociaux aux nécessiteux, ils considèrent que les mégastructures de l'État « are not helpful in providing meaning and identity for individual existence. Meaning, fulfillment, and personal identity are to be realized in the private sphere $»^{7}$ (Berger and Neuhaus, 2000, p. 144). À propos des structures de médiation, ils écrivent que cela n'implique pas de démanteler l'État providence, mais de réfléchir sérieusement à la façon dont il remplit ses objectifs. Reconnaît-il, par exemple, l'importance des structures de médiation? Quelles sont les limites de la capacité de l'État à offrir aux citoyens ou aux immigrants un sens à leur existence ? Ce n'est qu'une fois reconnues les limites de l'État providence, à la fois en termes de ressources et de sens, qu'il devient possible de réévaluer les théories et les pratiques afin d'intégrer la contribution des entités non étatiques à l'acculturation des immigrants et à leur intégration dans leur nouvelle société.

Je soutiens ici que l'immigration - en particulier celle de sans-papiers ou de demandeurs d'asile comme les Haïtiens en France - constitue un défi à l'État dans sa capacité à définir qui appartient ou non à la communauté politique. Comment l'idéologie républicaine peut-elle fonctionner comme modèle pour des immigrants qui, 
comme les Haïtiens, ont si peu de filières légales pour immigrer en France, et qui parviennent néanmoins à s'y établir? Les Haïtiens, comme d'autres immigrants venus des anciennes colonies françaises, sont amenés à quitter leur propre pays pour s'établir en France, même s'ils savent que les Français les ont réduits en esclavage quelques siècles plus tôt, et peuvent donc être tout à fait sceptiques sur le modèle républicain.

Ma recherche sur les immigrants haïtiens en France combine théorie et données chiffrées avec plusieurs mois de travail ethnographique. Au printemps 2003, j'ai interrogé 30 immigrés haïtiens qui fréquentaient la mission catholique haïtienne à Paris, ainsi que les responsables de sept associations laïques. Pendant cette période, j'ai assisté aux activités du week-end et en semaine de cette paroisse, ainsi qu'aux activités financées par les associations laïques haïtiennes en France. J'ai mené cette recherche en français et en créole haïtien. J'ai également rassemblé des données détaillées sur l'immigration haïtienne en France à partir de quatre sources : 1) une extraction de données demandée au bureau français du recensement, l'INSEE, chiffres pour la plupart encore inédits ; 2) la Direction de la Population et des Migrations du Ministère de l'Emploi et de la Solidarité ; 3) l'office des migrations internationales de la République française, Service des statistiques, des études et de la communication; 4) l'office français de la protection des réfugiés et apatrides (OFPRA).

\section{Les migrations haïtiennes en France}

Les liens coloniaux entre Haïti et la France ont entraîné quelques mouvements de population au cours des siècles. Les premiers Haïtiens commencèrent à arriver en France, fuyant les régimes de François et Jean-Claude Duvaliers, qui gouvernèrent en dictateurs sur Haïti de 1957 à 1986. Cependant, jusqu'aux années 1970, la plupart des Haïtiens vivant en France étaient étudiants ou exerçaient une profession libérale (Bastide, 1974). La première vague d'immigrants haïtiens était issue des classes supérieure et moyenne supérieure d'Haïti. Ils étaient, pour la plupart, concentrés autour de Port-au Prince, la capitale. Ils ne considéraient pas la France comme leur nouvelle patrie, mais étaient plutôt dans l'attente de retourner en Haïti dès que possible, à la faveur d'un régime plus démocratique. Beaucoup d'Haïtiens de cette première vague fondèrent des associations - en particulier des associations politiques - destinées à promouvoir le changement en Haïti, bien que très peu de ces associations aient survécu jusqu'à aujourd'hui (Alexis, 1998a).

Beaucoup des Haïtiens de cette vague initiale retournèrent en Haïti, en particulier lorsque Jean-Claude Duvalier se réfugia en France et qu'Haïti entama sa transition démocratique. Bien qu'il soit difficile de donner des chiffres précis et fiables, il semble que de nombreux Haïtiens qui firent leurs études en France au cours des années 1960 et 1970 émigrèrent au Canada et aux États-Unis, où ils trouvèrent de meilleures possibilités d'emploi, où ils étaient plus près d'Haïti, et pouvaient retrouver de plus vastes communautés d'expatriés, particulièrement à New-York, Montréal, et, plus récemment, Miami. L'accroissement de l'émigration haïtienne coïncida avec la décision française, en 1974, de mettre fin à toute migration du travail (Weil, 1995). En dépit de ce changement de politique, et alors que les conditions économiques et politiques en Haïti se détérioraient sous Baby Doc (1971-1986), l'émigration haïtienne s'accéléra, en particulier vers l'Amérique du Nord, mais aussi vers la France. On sait peu de choses sur cette seconde vague d'émigration vers la France. Les quelques études sur les Haïtiens en 
France publiées à ce jour (Alexis, 1998b ; Alexis, 1999 ; Bastide, Morin et Raveau, 1974 ; Delachet-Guyon, 1996) s'appuyaient sur des sondages auxquels répondirent quelques centaines de personnes, et ne présentaient que peu de données des recensements et statistiques sur l'immigration.

Les chiffres publiés à partir des recensements français ne fournissent que peu d'informations sur les Haïtiens à Paris. Comme le montre le tableau 1, la plupart sont entrés en France dans les années 1980. Ces chiffres ne concernent que ceux qui ne sont pas citoyens français. Entre 1982 et 1990, la population de Haïtiens en France est passée de 4724 à 12 311, soit un accroissement de $161 \%$. La croissance se ralentit au cours des années 1990, passant de 12311 à 15666 en 1999, soit un taux de croissance de $27 \%$.

Tableau 1 : Évolution du nombre d'immigrants haïtiens en France (sauf les naturalisés français)

\begin{tabular}{|l|l|l|l|l|l|}
\hline & Total & Hommes & Femmes & Croissance totale & Taux de croissance \\
\hline 1982 & 4724 & 2344 & 2380 & & \\
\hline 1990 & 12311 & 5768 & 643 & 7587 & $161 \%$ \\
\hline \hline 1999 & 15666 & 7409 & 8257 & 3355 & $27 \%$ \\
\hline
\end{tabular}

Source : INSEE, recensements 1982, 1990, 1990, CD-R6.

18 Afin d'obtenir des informations plus détaillées sur les Haïtiens en France, j'ai travaillé avec un statisticien de l'INSEE, pour extraire les données sur les Haïtiens du recensement de $1999^{9}$. En choisissant la catégorie «nationalité d'origine » comme unité primaire d'analyse dans le recensement français, j'ai pu identifier les personnes suivantes d'origine haïtienne : 1) citoyens haïtiens résidant en France; 2) personnes d'origine haïtienne naturalisées françaises ; 3) citoyens haïtiens nés en France. À partir de cette notion d'" origine haïtienne ", le tableau 2 montre qu'en 1999, on recensait près de 25000 Haïtiens vivant en France. Selon la loi française, les enfants nés en France de parents étrangers doivent choisir leur nationalité à 18 ans, ce qui fait que certains citoyens haïtiens vivant en France sont aussi nés en France (23,3\% de tous les Haïtiens), d'autres ont choisi la nationalité française.

Tableau 2 : Haïtiens en France, par nationalité de naissance et nationalité actuelle

\begin{tabular}{|l|l|l|}
\hline & Population & Pourcentage \\
\hline Nombre total de Haïtiens en France métropolitaine & 24911 & $100,0 \%$ \\
\hline Haïtiens naturalisés français & 9245 & $37,1 \%$ \\
\hline Citoyens haïtiens vivant en France & 9874 & $39,6 \%$ \\
\hline Citoyens haïtiens nés en France & 5792 & $23,3 \%$ \\
\hline
\end{tabular}

Source : INSEE, recensement de 1999. Extraction de l'auteure. 


\section{Les parcours migratoires des Haïtiens vers la France}

Bien que le tableau 2 indique le lieu de naissance et la nationalité des Haïtiens vivant en France, il ne nous dit pas comment ils ont pu immigrer. Une hypothèse est qu'ils immigrent d'abord dans les départements d'outre-mer (DOM) des Caraïbes. J'ai donc extrait les chiffres concernant les personnes d'origine haïtienne vivant en Guadeloupe, Martinique et en Guyane, que nous appellerons départements français d'Amérique (DFA). Les tableaux 3 et 4 montrent que, bien que le recensement de 1999 ait indiqué que 27349 Haïtiens vivent dans les DFA - soit à peine un peu plus qu'en France métropolitaine $-6,1 \%$ seulement des Haïtiens résidant en métropole ont indiqué qu'ils venaient des DFA.

Tableau 3 : Les Haïtiens dans les DFA

\begin{tabular}{|l|l|l|}
\hline & Total & Pourcentage \\
\hline Guyane & 15432 & $56,4 \%$ \\
\hline Guadeloupe & 10444 & $38,2 \%$ \\
\hline Martinique & 1470 & $5,4 \%$ \\
\hline Total des Haïtiens vivant dans les DFA & 27349 & $100 \%$ \\
\hline
\end{tabular}

Source : INSEE, recensement de 1999. Extraction de l'auteure.

Tableau 4 : Haïtiens dont le lieu de résidence est passé des DFA en 1990 à la France métropolitaine en 1999

\begin{tabular}{|l|l|}
\hline & Total \\
\hline Population haïtienne totale en France métropolitaine & 24 \\
911 \\
\hline Migrants haïtiens de Guyane vers la France métropolitaine & 1211 \\
\hline Migrants haïtiens de Guadeloupe vers la France métropolitaine & 215 \\
\hline Migrants haïtiens de Martinique vers la France métropolitaine & 89 \\
\hline $\begin{array}{l}\text { Total des migrants haïtiens des départements français d'Amérique vers la France } \\
\text { métropolitaine }\end{array}$ & 1515 \\
\hline $\begin{array}{l}\text { \% de Haïtiens en France qui ont migré des départementsfrançais d'Amérique vers la France } \\
\text { métropolitaine de } 1990 \text { à 1999 }\end{array}$ & $6,1 \%$ \\
\hline
\end{tabular}

Source : INSEE, recensement de 1999. Extraction de l'auteure. 
Avant d'analyser de façon plus détaillée les chiffres du recensement français sur le lieu de résidence, l'emploi et d'autres indicateurs socio-économiques concernant les Haïtiens, il est important de noter que ces chiffres peuvent ne pas refléter de façon exacte la population haïtienne en France. Dans la mesure où la politique française offre peu de voies d'immigration légale aux travailleurs haïtiens, beaucoup de responsables de la communauté haïtienne en France estiment que nombre d'entre eux arrivent en France avec un visa touristique, puis restent sur place sans papiers. Ces responsables estiment que le recensement sous-évalue d'environ $50 \%$ leurs effectifs. Ce seraient donc 50000 Haïtiens qui vivraient en France, et non les quelque 25000 recensés. Les responsables pensent qu'environ $20 \%$ des Haïtiens seraient sans papiers. Une étude détaillée confirme ces estimations. Delachet-Guyon (1996) a collecté les données des autorités nationales et locales, et calculé que plus de 30000 Haïtiens vivaient en France au début des années 1990, près du double de l'estimation du recensement officiel de 1990.

21 Une autre confirmation de l'estimation du nombre d'entrées clandestines de Haïtiens en France provient des données que j'ai collectées auprès des autorités françaises d'immigration, peu de Haïtiens ayant reçu un visa d'immigration en France. Afin de connaitre les types et le nombre des visas d'immigration de Haïtiens, je me suis rendue au centre de documentation de l'office de la Population et des Migrations du Ministère français de l'Emploi et de la Solidarité ${ }^{10}$. Chaque année, l'office publie le rapport Immigration et présence étrangère en France, qui précise combien de visas d'immigration en France sont donnés par nationalité. Avant 1993, les Haïtiens étaient classés dans la rubrique "Autres pays d'Amérique ». Je n'ai donc pu obtenir de données spécifiques que pour la période 1993-2001. Le tableau 5 montre qu'entre 1993 et 2001, peu de Haïtiens (5 133) sont entrés en France avec des visas de travail.

Tableau 5 : Sources officielles sur les migrations haïtiennes en France métropolitaine et dans les départements français d'Amérique, 1993-2001

\begin{tabular}{|l|l|l|l|l|l|l|}
\hline Année & Total & Salariés & $\begin{array}{l}\text { Travailleurs } \\
\text { non salariés }\end{array}$ & $\begin{array}{l}\text { Statut de } \\
\text { réfugiés }\end{array}$ & $\begin{array}{l}\text { Regroupement } \\
\text { familial }\end{array}$ & $\begin{array}{l}\text { Membres de } \\
\text { familles } \\
\text { françaises }\end{array}$ \\
\hline 1993 & 3208 & 1643 & 476 & 386 & 361 & 187 \\
\hline 1994 & 1927 & 910 & 193 & 283 & 309 & 148 \\
\hline 1995 & 1375 & 476 & 186 & 57 & 235 & 132 \\
\hline 1996 & 788 & 134 & 93 & 72 & 119 & 175 \\
\hline 1997 & 1865 & 139 & 200 & 72 & 158 & 159 \\
\hline 1998 & 1929 & 129 & 330 & 52 & 236 & 152 \\
\hline 1999 & 1360 & 56 & 69 & 61 & 342 & 104 \\
\hline 2000 & 1764 & 43 & 14 & 82 & 480 & 70 \\
\hline
\end{tabular}




\begin{tabular}{|l|l|l|l|l|l|l|}
\hline 2001 & 2087 & 37 & 5 & 210 & 517 & 97 \\
\hline $\begin{array}{l}\text { Total, } \\
1993-2001\end{array}$ & $\begin{array}{l}16 \\
303\end{array}$ & 3567 & 1566 & 1275 & 2757 & 1224 \\
\hline
\end{tabular}

Source : Immigration et Présence Étrangère en France, 1993-2001, André Lebon. La Documentation Française : Direction de la Population et des Migrations. Le Ministère de l'Emploi et de la Solidarité.

Tableau 5 (suite) : Sources officielles sur les migrations haïtiennes vers la France métropolitaine et les départements français d'Amérique, 1993-2001

\begin{tabular}{|c|c|c|c|c|c|}
\hline Année & Total & $\begin{array}{lr}\text { Membres } & \text { des } \\
\text { familles } & \text { de } \\
\text { réfugiés } & \end{array}$ & $\begin{array}{l}\text { «Cartes personnelles et } \\
\text { familiales " (yc asile } \\
\text { territorial)** }\end{array}$ & Visiteurs & $\begin{array}{l}\text { Bénéficiaires d'un } \\
\text { ré examen }\end{array}$ \\
\hline 1993 & 3208 & 57 & n.c. & 98 & n.c. \\
\hline 1994 & 1927 & 20 & n.c. & 64 & n.c. \\
\hline 1995 & 1375 & 17 & n.c. & 272 & n.c. \\
\hline 1996 & 788 & 22 & n.c. & 173 & n.c. \\
\hline 1997 & 1865 & 45 & n.c. & 283 & 809 \\
\hline 1998 & 1929 & 20 & n.c. & 237 & 773 \\
\hline 1999 & 1360 & 17 & $556(1)$ & 111 & 34 \\
\hline 2000 & 1764 & 22 & 1004 & 45 & 2 \\
\hline 2001 & 2087 & 37 & 1131 & 44 & 5 \\
\hline $\begin{array}{l}\text { Total, } \\
\text { 1993-2001 }\end{array}$ & $\begin{array}{l}16 \\
303\end{array}$ & 257 & 2135 & 1327 & 1623 \\
\hline
\end{tabular}

Source : Immigration et Présence Étrangère en France, 1993-2001, André Lebon. La Documentation Française : Direction de la Population et des Migrations. Le Ministère de l'Emploi et de la Solidarité.

Pourtant, à la différence des données du recensement, les chiffres du tableau 5 ne permettent pas de faire la différence entre les Haïtiens qui ont immigré en France métropolitaine et ceux qui l'ont fait dans les DFA. Puisque je m'intéressais au nombre de Haïtiens qui avaient reçu des visas pour la France métropolitaine, j'ai ensuite consulté les données du département Statistiques de l'office français des migrations internationales (OIM), qui indique où s'établissent les nouveaux immigrants haïtiens. Le tableau 6 montre que, au cours des deux années au cours desquelles le plus de visas de travail ont été donnés à des Haïtiens, plus de $90 \%$ avaient immigré dans les DFA. En 1993-1994, un très petit nombre - une centaine environ - avait reçu des visas pour Paris et sa banlieue. Étant donné le faible nombre de visas de travail délivré en France métropolitaine, je n'ai collecté que les informations pour 1993, 1994 et 2000. En 2000, 
seuls 9 Haïtiens ont immigré à Paris et sa banlieue, avec un visa permanent de travail salarié, ce qui indique très clairement que l'immigration pour travailler en France ne constitue pas une option envisageable pour les Haïtiens.

Tableau 6 : Entrée de travailleurs permanents de nationalité haïtienne par département

\begin{tabular}{|l|l|l|l|l|l|l|l|}
\hline Année & Total & Paris & Guadeloupe & Martinique & Guyane & Banlieue Paris & Autres dép. \\
\hline \hline 1993 & 1643 & 33 & 1286 & 1 & 226 & 88 & 9 \\
\hline 1994 & 910 & 31 & 38 & 3 & 784 & 49 & 5 \\
\hline \hline 2000 & 56 & 4 & 32 & 0 & 14 & 5 & 1 \\
\hline
\end{tabular}

Source : OMISTATS, Annuaire des Migrations 1993, 1994, et 2000. Office des Migrations Internationales (OMI). La Documentation Française.

23 Si aussi peu de Haïtiens viennent en France avec des visas de travail, quelles sont les autres possibilités légales? Très peu immigrent en tant que réfugiés. Le tableau 5 cidessus montre qu'en dépit des nombreux soulèvements politiques en Haïti, en particulier le coup d'État de 1991-1994, seuls 1532 Haïtiens et leur famille sont arrivés en France en tant que réfugiés entre 1993 et 2001.

Sans surprise, après les visas de travail, le principal mode d'entrée des Haïtiens en France est celui du regroupement familial (2 757) selon le tableau 5, suivi par l'obtention de la carte personnelle et familiale (2 135). Créée en 1999, elle confère un droit de résidence temporaire aux immigrants déjà sur le territoire français, et qui ont une raison majeure pour ne pas retourner dans leur pays (en dehors de la crainte d'une persécution politique, qui leur donnerait le droit à l'asile politique). Bien que cette loi ne date que de 1999, ce fut, entre 1999 et 2001, de loin, le principal motif de migration légale d'Haïti en France.

25 J'ai souhaité ensuite examiner dans quelle catégorie étaient rangés les Haïtiens bénéficiaires de la carte personnelle et familiale : époux et parents de citoyens français (649), liens personnels et familiaux (615), résidence en France depuis 10 à 15 ans (593), et mineurs ayant vécu en France depuis l'âge de 10 ans.

Tableau 7 : Haïtiens en possession d'une carte personnelle et familiale

\begin{tabular}{|l|l|lr|l|l|l|l|}
\hline Année & Total & $\begin{array}{l}\text { Époux et } \\
\text { Parents de } \\
\text { citoyens } \\
\text { français }\end{array}$ & $\begin{array}{l}\text { Époux de } \\
\text { chercheurs } \\
\text { scientifiques }\end{array}$ & $\begin{array}{l}\text { Mineurs en } \\
\text { France } \\
\text { depuis l'âge } \\
\text { de 10 ans }\end{array}$ & $\begin{array}{l}\text { Liens } \\
\text { personnels et } \\
\text { familiaux }\end{array}$ & $\begin{array}{l}\text { Résidence en } \\
\text { France depuis } \\
10-15 \text { ans }\end{array}$ \\
\hline 2000 & 1004 & 274 & 1 & 149 & 282 & 298 \\
\hline 2001 & 1131 & 375 & 2 & 126 & 333 & 295 \\
\hline
\end{tabular}




\begin{tabular}{|l|l|l|l|l|l|l|}
\hline $\begin{array}{l}\text { Total, } \\
\text { 2000-2001 }\end{array}$ & 2135 & 649 & 3 & 275 & 615 & 593 \\
\hline
\end{tabular}

Source : Immigration et Présence Étrangère en France, 1993-2001, André Lebon. La Documentation Française : Direction de la Population et des Migrations. Le Ministère de l'Emploi et de la Solidarité.

Bien que la carte personnelle et familiale ait permis à plusieurs milliers de Haïtiens de régler provisoirement le problème de leur statut, elle ne constitue pas une carte de résidence permanente.

Afin de savoir combien de Haïtiens déjà en France ont demandé le droit d'asile, j'ai consulté l'Office français de protection des réfugiés et apatrides (OFPRA). Le tableau 8 montre que le nombre total de décisions concernant les Haïtiens demandeurs d'asile entre 1981 et 2002 s'élevait à près de 23000 , c'est-à-dire à peu près autant que le nombre de Haïtiens recensés en 1999. Près de 18000 décisions, soit $78 \%$ du total, ont été rejetées. De plus, le taux d'octroi de l'asile, pour les Haïtiens, a très fortement diminué au cours des années récentes. Depuis 1995, ce taux est passé en dessous de $20 \%$; au cours de l'année la plus récente disponible, 2001, ce taux d'acceptation fut de $3,3 \%$ - en réalité de $7,1 \%$ en raison du succès des procédures d'appel. Comme la situation politique et économique en Haïti ne s'est pas améliorée, on peut présumer que la plupart des demandeurs d'asile - et de leur famille - qui ont été rejetés sont restés en France. À la lumière des chiffres de l'OFPRA, le chiffre de 25000 Haïtiens en France semble très bas, si l'on considère que 18000 Haïtiens ont vu leur demande d'asile rejetée au cours des deux dernières décennies.

Tableau 8 : Demandeurs d'asile Haïtiens en France, 1981-2001

\begin{tabular}{|l|l|l|l|l|l|l|l|}
\hline Année & $\begin{array}{l}\text { Première } \\
\text { demande }\end{array}$ & Décisions & Accords & $\%$ & Rejets & $\begin{array}{l}\text { Accord après } \\
\text { invalidation* }\end{array}$ & $\begin{array}{l}\text { T } \\
\text { d'accords* }\end{array}$ \\
\hline 1981 & 562 & 834 & 490 & $58,8 \%$ & 344 & & \\
\hline 1982 & 296 & 433 & 388 & $\mathbf{8 9 , 6} \%$ & 45 & & \\
\hline 1983 & 1047 & 569 & 348 & $\mathbf{6 1 , 2} \%$ & 221 & & \\
\hline 1984 & 1015 & 1104 & 424 & $38,4 \%$ & 680 & & \\
\hline 1985 & 635 & 1127 & 376 & $33,4 \%$ & 751 & & \\
\hline 1986 & 569 & 1053 & 310 & $29,4 \%$ & 743 & & \\
\hline 1987 & 648 & 601 & 82 & $13,6 \%$ & 519 & & \\
\hline 1988 & 1450 & 1120 & 285 & $25,4 \%$ & 835 & & \\
\hline 1989 & 2240 & 1890 & 186 & $9,8 \%$ & 1704 & & \\
\hline 1990 & 794 & 1827 & 269 & $\mathbf{1 4 , 7} \%$ & 1558 & & \\
\hline
\end{tabular}




\begin{tabular}{|l|l|l|l|l|l|l|l|}
\hline 1991 & 577 & 962 & 260 & $27,0 \%$ & 702 & & \\
\hline 1992 & 567 & 297 & 520 & $22,6 \%$ & 1777 & & \\
\hline 1993 & 301 & 1460 & 386 & $26,4 \%$ & 1074 & & \\
\hline 1994 & 366 & 961 & 283 & $29,4 \%$ & 678 & & \\
\hline 1995 & 146 & 681 & 57 & $\mathbf{8 , 4} \%$ & 624 & & \\
\hline 1996 & 138 & 438 & 72 & $\mathbf{1 6 , 4} \%$ & 366 & & \\
\hline 1997 & 108 & 396 & 72 & $\mathbf{1 8 , 2} \%$ & 324 & & \\
\hline 1998 & 357 & 266 & 52 & $\mathbf{1 9 , 5} \%$ & 214 & & \\
\hline 1999 & 503 & 518 & 61 & $\mathbf{1 1 , 8} \%$ & 457 & & \\
\hline 2000 & 1873 & 1457 & 82 & $\mathbf{5 , 6} \%$ & 1375 & & \\
\hline 2001 & 2713 & 2946 & 97 & $\mathbf{3 , 3} \%$ & 2849 & 113 & \\
\hline Total & 16905 & 2240 & 5100 & $\mathbf{2 2 , 2} \%$ & 17840 & & \\
\hline
\end{tabular}

* Rejets après invalidation par la Commission de recours.

Source : OFPRA, Office Français de la Protection des Réfugiés et Apatrides.

28 Une autre voie d'entrée légale possible de Haïti en France - celle du visa étudiant apparaît également plus restreinte que prévu. De 1993 à 2001, seuls 1089 Haïtiens ont obtenu des visas d'études en France.

Tableau 9 : Haïtiens entrés en France avec un statut temporaire d'étudiant

\begin{tabular}{|l|l|}
\hline Année & \\
\hline 1993 & 71 \\
\hline 1994 & 88 \\
\hline 1995 & 96 \\
\hline 1996 & 182 \\
\hline 1997 & 159 \\
\hline 1998 & 200 \\
\hline 1999 & 105 \\
\hline 2000 & 93 \\
\hline
\end{tabular}




\begin{tabular}{|l|l|}
\hline 2001 & 95 \\
\hline Total, 1993-2001 & 1089 \\
\hline
\end{tabular}

Source : Immigration et Présence Étrangère en France, 1993-2001, André Lebon. Direction de la Population et des Migrations. Le Ministère de l’Emploi et de la Solidarité. La Documentation Française.

Dans leur ensemble, les chiffres sur les entrées et régularisations de Haïtiens en France montrent que seule une faible partie d'entre eux est arrivée par des voies légales et permanentes. Comme de nombreux Haïtiens ont vu leur demande d'asile rejetée, ou tentent de régulariser leur statut via la carte personnelle et familiale, ils conservent un statut légal précaire.

\section{Profil socio-économique et localisation sociale à partir du recensement de 1999}

Les tableaux 10 à 14 ci-après reviennent sur les données extraites du recensement de 1999. L'image démographique de la population haïtienne qui ressort de ces tableaux est celle d'une population jeune, récemment arrivée, dans une position économique faible à moyenne, avec une majorité de familles biparentales. Bien qu'ayant, dans l'ensemble, un niveau d'éducation très moyen, avec une minorité significative de personnes possédant un niveau élevé de formation, peu de Haïtiens travaillent, en France, dans les professions libérales.

Le tableau 10 montre qu'en Ile-de-France, seuls $10 \%$ des Haïtiens habitent Paris; les autres vivent en banlieue. Ils se concentrent d'abord en Seine-Saint-Denis (6 787 individus, soit $30 \%$ de la population haïtienne en Ile-de-France). Trois autres départements connaissent de fortes concentrations de Haïtiens: le Val-d'Oise (4160, soit $18,9 \%$ ), les Hauts-de-Seine (3138, soit 14,3 \%) et le Val-de-Marne (2617, soit 11,9 \%).

Tableau 10 : Départements de résidence des Haïtiens vivant

en Île-de-France

\begin{tabular}{|l|l|l|}
\hline & Total & Pourcentage \\
\hline Total, Haïtiens en Île-de-France & 22000 & $100,0 \%$ \\
\hline \hline Seine-Saint-Denis & 6787 & $30,9 \%$ \\
\hline \hline Val-d'Oise & 4160 & $18,9 \%$ \\
\hline Hauts-de-Seine & 3138 & $14,3 \%$ \\
\hline Paris & 2698 & $12,3 \%$ \\
\hline Val-de-Marne & 2617 & $11,9 \%$ \\
\hline \hline Essonne & 1069 & $4,9 \%$ \\
\hline
\end{tabular}




\begin{tabular}{|l|l|l|}
\hline Seine-et-Marne & 840 & $3,8 \%$ \\
\hline Yvelines & 691 & $3,1 \%$ \\
\hline
\end{tabular}

Source : INSEE, recensement de 1999. Extraction de l'auteure.

scolaire (moins de 19 ans). Ces chiffres montrent que la majorité des Haïtiens sont en âge de travailler, ou entreront sur le marché du travail dans quelques années. Les Haïtiens - comme d'autres groupes d'immigrés en France - ont une population proportionnellement plus jeune que la population en général (OCDE, 2002).

Tableau 12 : Haïtiens en France par tranche d'âge

\begin{tabular}{|l|l|l|}
\hline Âge & Population & Pourcentage \\
\hline Total & 24911 & $100,0 \%$ \\
\hline $0-19$ & 8262 & $33,2 \%$ \\
\hline $20-39$ & 9297 & $37,3 \%$ \\
\hline $40-59$ & 6556 & $26,3 \%$ \\
\hline $60-74$ & 623 & $2,5 \%$ \\
\hline $75+$ & 173 & $0,7 \%$ \\
\hline
\end{tabular}

Source : INSEE, recensement de 1999. Extraction de l'auteure.

Étant donné le faible niveau d'éducation en Haïti, les résultats des Haïtiens en France en matière d'éducation apparaissent relativement élevés. Cela révèle la probabilité d'une auto-sélection des migrants: ceux qui possèdent déjà un niveau moyen 
d'éducation ont peut-être une plus grande propension à émigrer en France que ceux qui n'ont pas ou peu de formation scolaire. D'après le recensement de 1999, plus de $77,6 \%$ des Haïtiens en France possédaient un niveau d'éducation supérieur à l'école primaire. La majorité d'entre eux possède un niveau d'instruction secondaire : 44,8 \% ont été au collège ou au lycée, et $20 \%$ ont le niveau baccalauréat. Un groupe relativement restreint $(12,7 \%)$ a une formation supérieure. En raison des faibles possibilités d'étudier à l'université en Haïti, ceux qui ont une formation universitaire l'ont probablement acquise en France.

Tableau 13 : Niveau d'éducation des Haïtiens en France

\begin{tabular}{|l|l|l|}
\hline & Population & Pourcentage \\
\hline Total de la population de 15 ans et plus & 19256 & $100,0 \%$ \\
\hline École primaire & 4318 & $22,4 \%$ \\
\hline École secondaire et professionnelle1 & 8629 & $44,8 \%$ \\
\hline Lycée2 & 3859 & $20,0 \%$ \\
\hline \hline Université3 & 2450 & $12,7 \%$ \\
\hline
\end{tabular}

1 Selon le recensement: Collège, classes de 6e à 3e, CAP, BEP.

2 Selon le recensement: Classes de seconde, première ou terminale.

3 Selon le recensement : Études Supérieures (facultés, IUT, etc.).

Source : INSEE, recensement de 1999. Extraction de l'auteure.

Comme l'indique le tableau 14, les Haïtiens qui travaillent en France appartiennent aux catégories ouvrière et employée du recensement. Ce sont les secteurs de l'économie qui connaissent les plus forts taux de chômage, ce qui peut expliquer en partie le taux de chômage élevé des Haïtiens en France de $28,4 \%$. Ce taux est plus de deux fois supérieur à celui des Français de souche $(12,5 \%, \mathrm{OCDE}, 2002)$. Un capital humain (éducation, expérience professionnelle, connaissance de la langue française) moins important peut en partie expliquer le taux élevé de chômage des étrangers haïtiens. Mais d'autres facteurs contextuels, tels que l'impossibilité d'obtenir le droit de résidence ou la citoyenneté, le manque de réseaux sur le marché du travail et les discriminations ethniques contribuent à expliquer les forts niveaux de chômage des étrangers en France (Simon, 2003). Alors qu'aux États-Unis, le travail indépendant pourrait être considéré comme une façon d'éviter le chômage, peu de Haïtiens en France (133 personnes, soit $1 \%$ de la population active totale haïtienne) ont indiqué travailler à leur compte - artisans, commerçants ou chefs d'entreprise.

Tableau 14 : Activité économique des Haïtiens en France

\begin{tabular}{|l|l|l|l|}
\hline & Total & Employés EAP & Chômeurs EAP \\
\hline Total & 13323 & 5124 & 2021 \\
\hline Agriculture & - & - & - \\
\hline
\end{tabular}




\begin{tabular}{|l|l|l|l|}
\hline Artisans, commerçants et chefs d'entreprise & 133 & 93 & 40 \\
\hline Professions libérales et éducation supérieure & 164 & 147 & 17 \\
\hline Professions intermédiaires & 464 & 378 & 86 \\
\hline Employés & 4685 & 3389 & 1296 \\
\hline Ouvriers & 1340 & 117 & 223 \\
\hline Retraités & 227 & - & - \\
\hline Sans activité professionnelle & 6310 & - & 359 \\
\hline
\end{tabular}

Source : INSEE, recensement 1999. Extraction de l'auteure.

Tableau 15 : Taux de chômage des Haïtiens en France

\begin{tabular}{|l|l|l|}
\hline & Total & Pourcentage \\
\hline Population active totale & 13319 & $100,0 \%$ \\
\hline Population active employée & 9542 & $71,6 \%$ \\
\hline Population active au chômage & 3777 & $28,4 \%$ \\
\hline
\end{tabular}

Source : INSEE, recensement de 1999. Extraction de l'auteure.

Comme le recensement français ne pose pas de question sur les revenus, j'ai évalué plusieurs indicateurs de statut socio-économique. D'autres indicateurs du statut économique du foyer sont disponibles dans le recensement, tels que le type de foyer et la propriété du logement. La majorité des Haïtiens en France, soit 53,7 \%, vit dans des foyers avec deux parents mariés. $20 \%$ seulement habitent des logements possédés par la famille; les autres vivent en location (voir en annexe, tableau C). Le tableau D en annexe montre que $32,1 \%$ des Haïtiens vivent dans des logements de 70 à $100 \mathrm{~m}^{2}$; $34,4 \%$ habitent des appartements d'une taille moyenne de 40 à $70 \mathrm{~m}^{2}$, et $17,6 \%$ dans des logements de moins de $40 \mathrm{~m}^{2}$.

\section{Les bases de l'insertion}

Une grande partie des débats sur l'insertion des immigrants en France est centrée sur les forces et les faiblesses du modèle d'intégration, centré sur l'idéologie républicaine et la laïcité. Les chiffres présentés ci-dessus font apparaître un problème que peuvent difficilement résoudre modèle républicain et laïcité : les inégalités économiques entre immigrants haïtiens et Français de souche. Je soutiens que, sans quelque amélioration de ces inégalités, il est difficile d'imaginer une intégration culturelle sans faille des immigrants dans la société française. En fait, la crise des banlieues démontre amplement à quel point les inégalités socio-économiques, ce que les Français 
pourraient appeler l'exclusion sociale, peuvent produire d'importants désordres sociaux.

Je me tourne à présent brièvement vers mon travail de terrain auprès des immigrants haïtiens, qui illustre une autre façon d'envisager l'insertion des immigrants. Ce travail montre comment ils arrivent en France avec des schèmes culturels forgés dans leur pays d'origine, et comment ils les utilisent pour construire des institutions - à la fois religieuses et civiles - pour les aider à s'adapter. En opposition totale avec le modèle républicain français, qui met l'accent sur les liens individuels à l'État, je considère que les croyances religieuses et les récits fournissent une forme de médiation culturelle - ou une source de sens - et que les associations religieuses et civiles sont des médiatrices institutionnelles entre les immigrants haïtiens et l'État français.

\section{La communauté catholique haïtienne de Paris}

40 En Haïti, comme dans beaucoup de pays en voie de développement, les structures de l'État et les institutions religieuses exercent une forte influence culturelle et jouent un rôle social important. À travers toute l'histoire d'Haïti, la religion - qu'il s'agisse du catholicisme, du protestantisme ou du vaudou - a eu un fort impact sur la société haïtienne (Nérestant, 1994). Au cours des années 1970, alors que l'immigration haïtienne en France s'accélérait, la théologie de la libération inspira de nombreux mouvements catholiques en Haïti, qui tentèrent de présenter des objectifs politiques et sociaux. Comme de nombreux responsables de mouvements de la théologie de la libération défiaient l'autorité des Duvalier, il n'est pas surprenant de retrouver quelques-uns de ces dirigeants de base et une partie du clergé en France.

41 Leur effectif en France augmentant au cours des années 1970, les immigrants haïtiens commencèrent à se réunir de façon informelle pour prier. Le groupe d'origine n'était constitué que de 25 personnes, sous la conduite d'un prêtre missionnaire français qui avait officié en Haïti. Quelques années plus tard, en 1982, l'archevêché de Paris a créé une mission destinée aux Haïtiens, la communauté haïtienne catholique de Paris. Celleci représente le principal regroupement de Haïtiens en France. C'est aussi le seul groupe de ce type ayant un clergé nommé pour diriger la communauté, bien que deux groupes plus restreints de catholiques haïtiens se réunissent également en banlieue pour la prière et la messe ${ }^{11}$.

42 La fondation de la communauté catholique haïtienne à Paris illustre l'une des façons dont les immigrants moyens s'efforcent de transposer leurs schèmes culturels dans un nouvel environnement institutionnel. L'idéologie républicaine est, pour une grande part, top down, car elle met l'accent sur ce que l'État fait pour les immigrants, mais les efforts bottom up devraient également faire partie de nos théories sur l'intégration des immigrés. Le concept de structures de médiation contribue à combler le vide entre les approches top down et bottom up. Afin de passer de schèmes culturels à la mise en place d'institutions, les immigrants doivent, de quelque façon, interagir avec l'environnement d'accueil. Dans le cas présent, ils l'ont fait avec l'aide de ce qui est peut-être la principale institution de la société civile en France, l'Église catholique.

Par rapport aux religions protestantes ou non-chrétiennes, les immigrants catholiques en France tirent certes bénéfice du vaste réseau d'organisations catholiques, mais l'environnement institutionnel en France limite aussi l'étendue des médiations que 
peuvent offrir ces organisations catholiques aux immigrants. J'ai constaté que le clergé et les dirigeants laïcs de la communauté catholique haïtienne à Paris étaient très préoccupés par les problèmes sociaux posés aux immigrants haïtiens, et pourtant plusieurs facteurs limitaient leur capacité à véritablement trouver des solutions. Par rapport aux autres groupes d'immigrants en France, et aux communautés haïtiennes au Canada et aux États-Unis, un nombre relativement faible de Haïtiens vit en France. Comme nous l'avons vu, ils sont dispersés dans toute la banlieue parisienne, et il n'y a donc aucune localité centrale favorisant leur unité. De plus, et à la différence d'Haïti, et même des Haïtiens au Canada et aux États-Unis, la communauté catholique haïtienne de Paris repose intégralement sur le volontariat de laïques. La communauté manque même de fonds pour rémunérer un vicaire à plein temps, et doit compter sur un prêtre haïtien qui étudie en France pour assurer les services religieux.

Bien que les structures institutionnelles du catholicisme haïtien en France semblent très fragiles, la foi apporte aux membres actifs de cette communauté les outils nécessaires pour produire un discours optimiste dans des circonstances difficiles. Pour beaucoup de Français, le modèle républicain et la laïcité fournissent un discours sur la façon dont les immigrés devraient s'adapter à la société française. Pourtant, les Haïtiens auxquels j'ai parlé n'utilisaient pas ce discours, mais plutôt leur croyance religieuse pour créer leur propre discours sur leurs luttes en migration et pour l'intégration.

Les personnes que j'ai rencontrées à la mission catholique haïtienne de Paris vivent en banlieue, ont des emplois faiblement rémunérés, et considèrent que les Français ne les traitent pas d'égal à égal. Ils se tournent vers leur communauté religieuse pour y trouver dignité, identité et communauté. Lorsqu'ils assistent à des manifestations ou participent à des programmes sociaux de la communauté, les Haïtiens parlent de leur crainte d'être arrêtés et de devoir présenter des papiers officiels. Ils discutent aussi des difficultés à trouver un travail et un logement, à élever leurs enfants.

D'autres formes d'organisation laïques pourraient aussi fournir aux Haïtiens des lieux de rassemblement. Pourtant leur croyance religieuse leur apporte un cadre culturel irremplaçable, qu'ils utilisent en général pour produire leurs récits d'espoir. Au cours de mes entretiens, les Haïtiens que je rencontrais fréquemment décrivaient cette forte proximité avec Dieu avec des expressions comme «être baigné dans la foi ». Face à de grandes difficultés, beaucoup me disaient se tourner vers la prière. Ainsi, une femme me dit : " Jésus est mon modèle. Il est mon meilleur ami. Je ne suis rien sans Jésus. Je lui parle toute la journée, juste comme je vous parle maintenant ». La foi et la participation à une mission catholique à caractère ethnique devient une stratégie pour produire des récits d'auto-valorisation. Ainsi, durant les services à la mission catholique, les lectures de la Bible et les sermons illustrent le fait que les Chrétiens accomplissent, dans ce monde, " un pèlerinage plein de larmes ». Les chants religieux se lamentent sur la douleur et la perte, tout en professant la foi en un Dieu bon. En d'autres termes, le modèle républicain promet la fraternité, l'égalité et la solidarité, mais les croyances et les pratiques religieuses des Haïtiens leur apportent un schème culturel qui reconnaît que ce type d'objectif est le plus souvent atteint à travers des souffrances temporaires. 


\section{L'association développement d'Haïti} interrogé beaucoup de ses membres, mais j'ai aussi enquêté sur les associations haïtiennes à Paris. Bien que les Haïtiens aient fondé quelque 300 associations, j'ai constaté que la plupart n'existent que sur le papier, et qu'au mieux 40 d'entre elles sont vraiment actives. Parmi elles, environ 25 développent des projets en Haïti plutôt qu'en France (Glaude, 2001). Les quelque 10 associations haïtiennes qui financent des activités en France s'occupent principalement d'aider les immigrants dans trois domaines : 1) obtenir des papiers officiels ; 2) trouver du travail ; 3) trouver un logement abordable et adapté. Malgré les efforts importants déployés par leurs responsables, ces associations n'ont en général que peu ou pas de ressources et doivent reposer entièrement sur le volontariat. Bien que les responsables avec lesquels j'ai discuté aient tous exprimé des doutes sur leur propre capacité à aider efficacement à l'intégration des Haïtiens, l'existence de ce type d'associations souligne à nouveau l'importance des structures de médiation dans l'intégration des immigrants.

Ce n'est pas un effet du hasard si les seules associations qui sont parvenues à travailler directement à la fois avec des Haïtiens et avec les autorités françaises sont en relation étroite avec la communauté catholique des Haïtiens de Paris. L'un des fondateurs salariés de cette communauté, M. René Benjamin, a créé Développement d'Haïti en 1961 pour encourager les étudiants haïtiens en France à participer au développement d'Haïti à leur retour. Mais, alors que de plus en plus de Haïtiens des classes laborieuses commencèrent à arriver en France à partir des années 1970, M. Benjamin a transformé les objectifs de son mouvement afin d'aider à leur intégration. Bien que, dans leur majorité, les membres de la communauté catholique haïtienne de Paris soient des volontaires, la communauté religieuse est le seul endroit où j'ai pu observer le travail commun de salariés, comme M. Benjamin, et d'immigrants volontaires, tous haïtiens, sur une base régulière. Ainsi, cette communauté religieuse aide-t-elle à combler le fossé de classe entre Haïtiens en France.

La principale priorité de Développement d'Haïti consiste en un type de médiation directe. M. Benjamin et sa petite équipe aident chaque mois environ 150 Haïtiens à demander la régularisation de leur statut, soit comme demandeurs d'asile, soit, plus récemment, par le biais de la carte personnelle et de vie familiale. Comme il ressort des tableaux ci-dessus, de nombreux Haïtiens en France n'ont pas de papiers officiels. Pour se sortir de cette situation, ils reposent souvent sur un membre de leur propre communauté, auquel ils font confiance pour les aider à agir auprès de l'État français. En fournissant à Haïti Développement des fonds pour aider les demandeurs d'asile à remplir les papiers nécessaires, l'État français reconnaît la nécessité de ce type de médiation. En plus des financements publics, la mission catholique de France apporte un soutien technique et financier à Haïti Développement, en créant des programmes d'apprentissage du français, de prévention du sida, de communication familiale et des cours de cuisine. C'est en partie parce qu'elle a trouvé des fonds pour ses programmes que Haïti Développement est la seule association haïtienne en France qui possède un siège permanent, des bureaux aux heures ouvrables et un personnel rémunéré.

Une partie du succès de Haïti Développement vient aussi de la position de M. Benjamin comme responsable de la communauté catholique haïtienne de Paris. Sa participation à une communauté religieuse lui permet de nouer, avec les nouveaux immigrants, des

Revue européenne des migrations internationales, vol. 24 - $n^{\circ} 1$ | 2008 
liens qui engendrent confiance et solidarité. L'exemple de Haïti Développement montre que les structures de médiation comportent des éléments à la fois culturels et institutionnels. Les récits produits à travers la pratique religieuse constituent un premier pas vers la fin de l'exclusion sociale et Haït Développement établit le lien entre la communauté haïtienne et les institutions françaises. Même si le principe de laïcité interdit la plupart des aides directes à des organisations religieuses, les responsables des organisations laïques d'immigrés peuvent fort bien trouver légitimité et fondement de la confiance qui leur est faite dans leurs activités religieuses. Comme M. Benjamin participe à la pratique religieuse des Haïtiens, en même temps qu'il traite directement avec les autorités françaises, il est un médiateur plus efficace que d'autres Haïtiens qui travaillent exclusivement dans le domaine civil.

\section{Conclusion}

51 Comme la plupart des Haïtiens sont arrivés au cours des 30 dernières années, il est trop tôt pour estimer la réussite ou l'échec de l'intégration. C'est pourquoi je me suis centrée sur les premières étapes du processus, comme par exemple l'obtention de papiers ou d'un logement et d'un emploi. Les chiffres que j'ai présentés démontrent que, d'une façon proche de celle d'autres immigrants résidant dans les banlieues françaises, les Haïtiens sont confrontés à l'exclusion sociale. En premier lieu, $20 \%$ d'entre eux sont sans papiers, et seulement $33 \%$ ont la nationalité française. Ensuite, et bien que beaucoup aient un niveau d'éducation moyen, voire élevé, ils connaissent des taux importants de chômage - près de $30 \%$. En troisième lieu, ils vivent dans des banlieues de Paris qui sont stratifiées selon la racialisation des populations et leur origine sociale. En conséquence, ils n'ont que des contacts directs limités avec les institutions françaises.

Les associations haïtiennes - à la fois religieuses et civiles - s'efforcent d'établir des médiations entre immigrants haïtiens et autorités françaises, mais sans grand succès. Pendant la durée de ma recherche, la Communauté catholique haïtienne de Paris a été la seule organisation réunissant schémas culturels et ressources institutionnelles qui constituait une médiation efficace entre immigrants haïtiens et autorités françaises. Alors que le débat se poursuit sur le modèle français d'intégration, je suggère d'accorder plus d'attention aux schèmes culturels des immigrants, ce qui pourrait fournir de nouvelles modalités d'interaction entre immigrants et État, permettant de mettre en oeuvre les objectifs d'intégration sociale et de mobilité socio-économique. L'adhésion au modèle républicain peut continuer à représenter un objectif d'intégration des immigrants, mais il faudrait accorder plus d'attention à leur position socio-économique alors qu'ils entreprennent leur parcours d'adaptation, et à la façon dont ils constituent des associations d'aide à leur intégration sociale.

Pour dépasser l'idéologie du modèle républicain français, il faut reconnaître les limites de la capacité de l'État à fournir du sens. Les structures de médiation contribuent à l'intégration des immigrants à la fois parce qu'elles créent un espace où les individus se fabriquent $d u$ sens et parce qu'elles fournissent une base institutionnelle grâce à laquelle les immigrants ordinaires peuvent entrer en contact avec l'État. Reconnaître que les immigrants créent des structures de médiation ne signifie pas abandonner les objectifs du modèle républicain et de la laïcité, mais c'est plutôt porter une attention soutenue aux immigrants eux-mêmes comme agents de leur propre intégration. 


\section{BIBLIOGRAPHIE}

ALEXIS Sergio (1998a) Typologie historique de la migration haïtienne en France (première partie), Diaforama, pp. 30-31.

ALEXIS Sergio (1998b) Typologie historique de la migration haïtienne en France (seconde partie), Diaforama, pp. 48-49.

ALEXIS Sergio (1999) Communauté Haïtienne en Île de France : une approche anthropologique nouvelle, Diaforama, pp. 30-32.

BASTIDE Roger, MORIN Françoise et RAVEAU François (1974) Les Haïtiens en France, Éditions Mouton, Paris.

BERGER Peter, NEUHAUS and RICHARD John (2000) To Empower People: From State to Civil Society, pp. 143-182 in The Essential Civil Society Reader: The Classic Essays, Don E. Eberly (éd), Lanham, MD: Rowman and Littlefield.

DELACHET-GUILLON Claude (1996) La communauté haïtienne en Île de France, L'Harmattan, Paris. ESPING-ANDERSEN Gosta (1990) Three Worlds of WelfareT Capitalism, Polity Press, Cambridge, UK.

GLAUDE Smith (2001) « Le dynamisme associatif des migrants haïtiens en France métropolitaine ». Mastère en Administration Économique et Sociale,Saint-Denis : Université Paris 8.

GORDON Milton Myron (1964) Assimilation in American Life: The Role of Race, Religion, and National Origins, Oxford University Press, New York.

HANDLIN Oscar (1951) The Uprooted: The Epic Story of the Great Migrations that Made the American People, Little Brown, Boston.

HOROWITZ Donald L. (1992) "Immigrants in Two Democracies: French and American Experience" pp. 3-35 in Immigrants in Two Democracies: French and American Experience, Donald L. Horowitz et Gérard Noiriel (éds), New York University Press, New York.

KASTORYANO Riva (1996) La France, l'Allemagne, et leurs immigrés : négocier l'identité, Armand Colin, Paris.

LAMONT Michèle (2000) The Dignity of Working Men: Morality and the Boundaries of Race, Class and Immigration, Harvard University Press, Cambridge, MA.

LEBON André (2001) Immigration et présence étrangère en France 1999: Premiers enseignements du recensement, La Documentation Française, Paris.

LIEBERMAN Robert (2001) "A Tale of Two Countries: The Politics of Color-Blindness in France and the United States." French Politics Culture and Society 19: 32-59.

NERESTANT Micial M. (1994) Religions et politique en Haïti, Éditions Karthala, Paris.

THOMAS William Isaac and ZNANIECKI Florian (1927), The Polish Peasant in Europe and America, Alfred A. Knopf, New York.

WIHTOL DE WENDEN Catherine (1998) "How Can One Be Muslim? The French Debate on Allegiance, Intrusion and Transnationalism." International Review of Sociology 8: 275-288. 


\section{ANNEXES}

Tableau A : Les Haïtiens par région en France

\begin{tabular}{|c|c|c|}
\hline Région & Population & Pourcentage \\
\hline Total & 24911 & $100,00 \%$ \\
\hline Île-de-France & 22000 & $88,31 \%$ \\
\hline Champagne-Ardenne & 44 & $0,18 \%$ \\
\hline Picardie & 223 & $0,90 \%$ \\
\hline Haute-Normandie & 97 & $0,39 \%$ \\
\hline Centre & 188 & $0,75 \%$ \\
\hline Basse-Normandie & 98 & $0,39 \%$ \\
\hline Bourgogne & 42 & $0,17 \%$ \\
\hline Nord-Pas-de-Calais & 76 & $0,31 \%$ \\
\hline Lorraine & 93 & $0,37 \%$ \\
\hline Alsace & 145 & $0,58 \%$ \\
\hline Franche-Comté & 44 & $0,18 \%$ \\
\hline Pays de la Loire & 443 & $1,78 \%$ \\
\hline Bretagne & 153 & $0,61 \%$ \\
\hline Poitou-Charentes & 81 & $0,33 \%$ \\
\hline Aquitaine & 104 & $0,42 \%$ \\
\hline Midi-Pyrénées & 124 & $0,50 \%$ \\
\hline Limousin & 25 & $0,10 \%$ \\
\hline Rhône-Alpes & 347 & $1,39 \%$ \\
\hline Auvergne & 56 & $0,22 \%$ \\
\hline Languedoc-Roussillon & 137 & $0,55 \%$ \\
\hline Provence-Alpes-Côte d'Azur & 385 & $1,55 \%$ \\
\hline
\end{tabular}




\begin{tabular}{|l|l|l|}
\hline Corse & 6 & $0,02 \%$ \\
\hline
\end{tabular}

Source : INSEE, recensement de 1999. Extraction de l'auteure.

Tableau B : Type de famille haïtienne en France

\begin{tabular}{|l|l|l|}
\hline & Total & Pourcentage \\
\hline Total & 24911 & $100,0 \%$ \\
\hline Foyers non familiaux & 3420 & $13,7 \%$ \\
\hline Familles monoparentales & 4814 & $19,3 \%$ \\
\hline Familles à deux parents mariés & 13376 & $53,7 \%$ \\
\hline Familles à deux parents non mariés & 2066 & $8,3 \%$ \\
\hline Familles à deux parents, autre statut marital & 1235 & $5,0 \%$ \\
\hline
\end{tabular}

Source : INSEE, recensement de 1999. Extraction de l'auteure.

Tableau C : Type de logement des Haïtiens en France

\begin{tabular}{|l|l|l|}
\hline & Population & Pourcentage \\
\hline Total & 24911 & $100,00 \%$ \\
\hline Pas de logement régulier & 578 & $2,32 \%$ \\
\hline Propriétaire & 4896 & $19,65 \%$ \\
\hline Locataire ou sous-locataire d'un appartement autre que HLM & 7676 & $30,81 \%$ \\
\hline Locataire ou sous-locataire d'un appartement HLM & 10618 & $42,62 \%$ \\
\hline Locataire ou sous-locataire d'un meublé ou d'un hôtel & 775 & $3,11 \%$ \\
\hline En logement libre & 368 & $1,48 \%$ \\
\hline
\end{tabular}

Source : INSEE, recensement de 1999. Extraction de l'auteure.

Tableau D : Taille des logements des Haïtiens en France

\begin{tabular}{|l|l|l|}
\hline & Total & Pourcentage \\
\hline \hline Total & 24911 & $100,0 \%$ \\
\hline
\end{tabular}




\begin{tabular}{|l|l|l|}
\hline Logement autre que traditionnel & 578 & $2,3 \%$ \\
\hline Moins de 40 mètres carrés & 4396 & $17,6 \%$ \\
\hline Entre 40 et 70 mètres carrés & 8564 & $34,4 \%$ \\
\hline Entre 70 et 100 mètres carrés & 7999 & $32,1 \%$ \\
\hline Entre 100 et 150 mètres carrés & 2750 & $11,0 \%$ \\
\hline Plus de 150 mètres carrés & 624 & $2,5 \%$ \\
\hline
\end{tabular}

Source : INSEE, recensement de 1999. Extraction de l'auteure.

\section{NOTES}

1. Sauf exception signalée, je ne me réfère ici qu'aux Haïtiens en France métropolitaine.

2. Les lecteurs intéressés par la comparaison internationale des structures de médiation et de l'intégration des immigrants pourront se référer à mon livre à paraître, dont le titre provisoire est Faith makes us Live: Comparing Haitian Immigrants in Three Countries.

3. « les identités raciales et ethniques... n'ont, en général, été considérées comme des catégories, ni politiquement, ni même statistiquement » (ndt).

4. Le principe de laïcité mis en œuvre dès l'époque de la Révolution française, cherchait à soumettre l'Église catholique à l'État. Il impliquait également l'interdiction d'une référence publique à l'appartenance religieuse. La séparation de l'Église et de l'État fut officiellement confirmée par la loi de 1905. Dans sa lignée, une loi interdisant le port ostensible de signes religieux dans l'enceinte scolaire est entrée en vigueur en 2004.

5. «La sous-société des immigrants constitue un élément médiateur entre la culture d'origine de l'immigrant et la culture américaine. Reconnaître ce fait est l'indispensable préalable à un fonctionnement effectif des moyens de communication et des réseaux d'influence qui, dans la vie des communautés d'immigrants, sont destinés à aider et à encourager la mise en place de véritables objectifs d'acculturation » (ndt)

6. « ces structares qui s'interposent entre la vie privée de l'individu et les grandes institutions de la vie publique » (ndt).

7. « ne parviennent pas à apporter sens et identité à l'existence de l'individu. Le sens, les réalisations, et l'identité personnelle se réalisent dans la sphère privée » (ndt).

8. Le père, François, était aussi appelé Papa Doc et le fils, Jean-Claude, était appelé Baby Doc.

9. Dimitri Bechaq, doctorant d'anthropologie à l'EHESS à l'époque, participa également à l'extraction des données.

10. Je voudrais remercier André Lebon de son aide pour m'avoir indiqué les sources qui m'étaient nécessaires.

11. Glaude (2001) estime qu'il existe au moins 40 Églises protestantes haïtiennes en France, avec entre 25 et 300 membres. Pour des raisons de temps, et parce que je m'intéressais à comparer la façon dont les institutions religieuses aident à l'adaptation des immigrants dans trois contextes nationaux, je n'ai conduit observation et entretiens que dans la principale communauté haïtienne catholique de Paris, et non dans les Églises protestantes et les cérémonies Vaudoues. 


\section{RÉSUMÉS}

Dans le présent article, j'utilise les chiffres officiels du gouvernement français et des entretiens avec des immigrants haïtiens pour analyser les voies que les Haïtiens ont utilisées pour entrer en France et s'y installer. Malgré l'absence de canaux légaux d'immigration des Haïtiens en France, le chiffre de la population haïtienne en France est passé, au cours des 20 dernières années, de quelques milliers d'immigrants, surtout dans les professions libérales, à 25_000_personnes de divers niveaux d'éducation et occupant des emplois le plus souvent faiblement qualifiés. En utilisant des statistiques encore inédites, recueillies à l'INSEE, je montre que la plupart des immigrés vivent en banlieue parisienne, et que, de façon peu différente d'autres résidants de banlieue, ils connaissent des taux de chômage et de pauvreté élevés. J'utilise ensuite des entretiens avec des Haïtiens en France et l'observation des évènements qui se produisent dans leur communauté pour discuter le fait que le débat en cours sur l'intégration des immigrés en France a largement sous-estimé le rôle potentiellement médiateur que les institutions religieuses peuvent jouer auprès des immigrés.

Mediating Structures and the Adaptation of Haitian Immigrants in Paris. In this article, I use official French government data and interviews with Haitian immigrants to analyze the paths through which Haitians have entered and settled in France. Despite the lack of legal channels for Haitian immigration to France, in the last 20 years, the size of the Haitian population in France has grown from just a few thousand mostly professional immigrants to include 25,000 people of varied educational levels and mostly low-wage occupations. Using never-before published data I collected from INSEE, I show that Haitian immigrants live mostly in the Parisian banlieue, and not unlike other residents of the banlieue, they exhibit high levels of unemployment and poverty. I then use interviews with Haitians in France and observations of their community events to argue that current debates about immigrant integration in France have largely overlooked the potential mediating role that religious institutions can play on behalf of immigrants.

Estructuras de mediación y de integración de inmigrantes haitianos en París. En este artículo, utilizo datos oficiales del gobierno francés y entrevistas con inmigrantes haitianos para analizar las trayectorias a través de las cuales los Haitianos han entrado y han colocado en Francia. A pesar de la carencia de los canales legales para la inmigración haitiana a Francia, en los 20 años pasados, el tamaño de la población haitiana en Francia ha crecido apenas de algunos mil, sobre todo inmigrantes profesionales, hasta 25.000 personas de niveles de enseñanza y principalmente empleos con bajo-salario. Usando datos nuevos nunca publicadas, recogídas del INSEE, muestro que los inmigrantes haitianos viven sobre todo en el banlieue parisiense, y no desemejante de otros residentes del banlieue, tienen altos niveles de desempleo y de pobreza. Entonces utilizo entrevistas con Haitianos en Francia y observaciones de sus acontecimientos de la comunidad para discutir que los discusiones actuales sobre la integración inmigrante en Francia han pasado por alto en gran parte el papel potencial de mediación que las instituciones religiosas saben desempeñar a los inmigrantes.

\section{INDEX}

Mots-clés : exclusion sociale, intégration sociale, médiation, pauvreté, stratégie migratoire, Haïtiens

Index géographique : France 


\section{AUTEURS}

\section{MARGARITA MOONEY}

Department of Sociology, University of North Carolina at Chapel Hill. 